\title{
Use of 3D Geometry Modeling of Osteochondrosis-like Iatrogenic Lesions as a Template for Press-and-Fit Scaffold Seeded with Mesenchymal Stem Cells
}

\author{
P. KRUPA ${ }^{1}$, P. KRŠEK ${ }^{2}$, M. JAVORNÍK ${ }^{3}$, O. DOSTÁL ${ }^{3}$, R. SRNEC ${ }^{4}$, D. USVALD ${ }^{5}$, \\ P. PROKS ${ }^{4}$, H. KECOVÁ ${ }^{4}$, E. AMLER ${ }^{6}$, J. JANČÁR ${ }^{7}$, P. GÁL $^{8}$, L. PLÁNKA ${ }^{8}$,
} A. NEČAS ${ }^{4}$

${ }^{1}$ Department of Medical Imaging and Radiology, St. Anne's University Hospital, Masaryk University, Brno, ${ }^{2}$ Faculty of Information Technology, University of Technology, Brno, ${ }^{3}$ Institute of Computer Science of Masaryk University, Brno, ${ }^{4}$ Department of Surgery and Orthopedics, Small Animal Clinic, Faculty of Veterinary Medicine, University of Veterinary and Pharmaceutical Sciences, Brno, ${ }^{5}$ Institute of Animal Physiology and Genetics, Academy of Sciences of the Czech Republic, Liběchov, ${ }^{6}$ Department of Biophysics, Second Faculty of Medicine, Charles University, Prague, ${ }^{7}$ Institute of Materials Chemistry, University of Technology, Brno, ${ }^{8}$ Department of Pediatric Surgery, Orthopedics and Traumatology, Masaryk University, Brno, Czech Republic

Received May 23, 2007

Accepted May 29, 2007

On-line available May 31, 2007

\begin{abstract}
Summary
Computed tomography (CT) is an effective diagnostic modality for three-dimensional imaging of bone structures, including the geometry of their defects. The aim of the study was to create and optimize 3D geometrical and real plastic models of the distal femoral component of the knee with joint surface defects. Input data included CT images of stifle joints in twenty miniature pigs with iatrogenic osteochondrosis-like lesions in medial femoral condyle of the left knee. The animals were examined eight and sixteen weeks after surgery. Philips MX 8000 MX and View workstation were used for scanning parallel plane cross section slices and Cartesian discrete volume creation. On the average, 100 slices were performed in each stifle joint. Slice matrices size was 512 x 512 with slice thickness of $1 \mathrm{~mm}$. Pixel (voxel) size in the slice plane was $0.5 \mathrm{~mm}$ (with average accuracy of $\pm 0.5 \mathrm{~mm}$ and typical volume size $512 \times 512 \times 100$ voxels). Three-dimensional processing of CT data and 3D geometrical modelling, using interactive computer graphic system MediTools formerly developed here, consisted of tissue segmentation (raster based method combination and $5 \%$ of manual correction), vectorization by the marching-cubes method, smoothing and decimation. Stifle- joint CT images of three individuals of different body size (small, medium and large) were selected to make the real plastic models of their distal femurs from plaster composite using rapid prototyping technology of Zcorporation. Accuracy of the modeling was $\pm 0.5 \mathrm{~mm}$. The real plastic models of distal femurs can be used as a template for developing custom made press and fit scaffold implants seeded with mesenchymal stem cells that might be subsequently implanted into iatrogenic joint surface defects for articular cartilage-repair enhancement.
\end{abstract}




\section{Key words}

Cartilage $\bullet$ CT imaging $\bullet$ Tissue segmentation $\bullet$ Joint resurfacing $\bullet$ Mesenchymal stem cells $\bullet$ Miniature pig

\section{Introduction}

Computed tomography (CT) and magnetic resonance (MR) imaging represent very helpful modalities used for imaging joint injuries and cartilage defects, in both clinical practice and research (Potter et al. 1998). Each of these techniques provides slightly different information, one complementary with the other. $\mathrm{CT}$ is the best modality for three-dimensional (3D) imaging of the bone structure and shape, and geometry of defects (Hall 1994). The MR is sensitive to changes of 3D density of hydrogen nucleus (Rubenstein et al. 1990), and can evaluate metabolic and chemical changes inside the tissues (MR spectroscopy) where structural changes are yet not visible (Kramer et al. 1992).

Standard CT/MR techniques of musculoskeletal system examination are based on 2D multiplanar images (coronal, sagittal, axial) (Burnstein et al. 2000). A modern trend is also volume rendering $3 \mathrm{D}$ visualization of the data (Recht et al. 1996, Disler et al. 1996). With developing technology of CT/MR images, 3D resolution of its data is better and it is possible to use it not only for $3 \mathrm{D}$ visualization of tissues but also for their 3D geometrical modelling (Krupa et al. 2004, Kršek et al. 2006). The 3D geometrical modelling is mathematical, vector based (by polygonal triangular mesh or by spline surfaces) description of particular tissue- boundary geometry. This technique is becoming more popular in surgery planning, simulations, navigations and training (Kršek et al. 2006, Černochová et al. 2005, Krupa et al. 2004), particularly in plastic surgery, stomatology, orthopedic surgery, traumatology, neurosurgery, etc. Formerly, we developed our own computer graphic system MediTools for 3D geometrical modelling of tissues, based on CT/MR data (Kršek et al. 2006, Černochová et al. 2005, Krupa et al. 2004). This system also makes it possible to create real plastic models of different body structures (joints, bones, vessels, etc.). Presently, this system is used in clinical practice at the Department of Medical Imaging and Radiology, St. Anne's University Hospital in Brno.

This work was aimed using the MediTools system for 3D geometrical modelling of distal femurs with iatrogenically created stifle joint surface defects in miniature pigs. The goal of the study was to optimize the creation of geometrical and real plastic models of the femoral component of the stifle joint. We evaluated the proposed process of model creation based on CT data retrieved from examinations of stifle joints in the animals. Results of this experimental work will be utilized in future research, which will be aimed at enhancement of cartilage repair with the use of mesenchymal stem cells (MSCs). Our future goal is to use the real plastic models of distal femurs to create individual press and fit collagen scaffold implants that could be (after seeding with mesenchymal stem cells) implanted into iatrogenic joint surface defects in our experimental work.

\section{Materials and methods}

\section{Experimental animals}

Twenty miniature pigs were included in the study. Iatrogenic osteochondrosis-like (OCD-like) lesions were created in the medial femoral condyle of the left stifle joints. All animals subsequently underwent CT examination under general anesthesia. During the whole study period, the animals were fed, handled and housed according to the principles of welfare. All procedures were carried out with the consent of the Ethical Committee (No. 46613/2003-1020).

\section{Anesthesia}

The miniature pigs were premedicated with a mixture of xylazine $(2 \mathrm{mg} / \mathrm{kg}$ body weight, SEDAZINE, Fort Dodge, USA), ketamine ( $2 \mathrm{mg} / \mathrm{kg}$ body weight, KETASET, Fort Dodge, USA) and zolazepam-tiletamine ( $2 \mathrm{mg} / \mathrm{kg}$ body weight, ZOLETIL 100, Virbac, France) intramuscularly. Anesthesia was induced by intravenous administration of propofol (1-2 mg/kg body weight, PROPOFOL, Fresenius, Austria) and then maintained with the mixture of oxygen, nitrous oxide (1:2) and isoflurane (FORANE, Abbott Laboratoires, France). All pigs were connected to a vital functions monitor (DATEX Cardiocap II). The logged values included heart rate $(\mathrm{HR})$, respiratory rate $(\mathrm{RR})$, mean arterial pressure (MAP), end-tidal $\mathrm{CO}_{2}$ concentration $\left(\mathrm{ETCO}_{2}\right)$ and hemoglobin saturation by oxygen $\left(\mathrm{SpO}_{2}\right)$. HR was sensed using a 3-terminal EKG with electrodes located on the patient's chest. MAP was measured using a disposable blood pressure transducer connected to a monitor after 


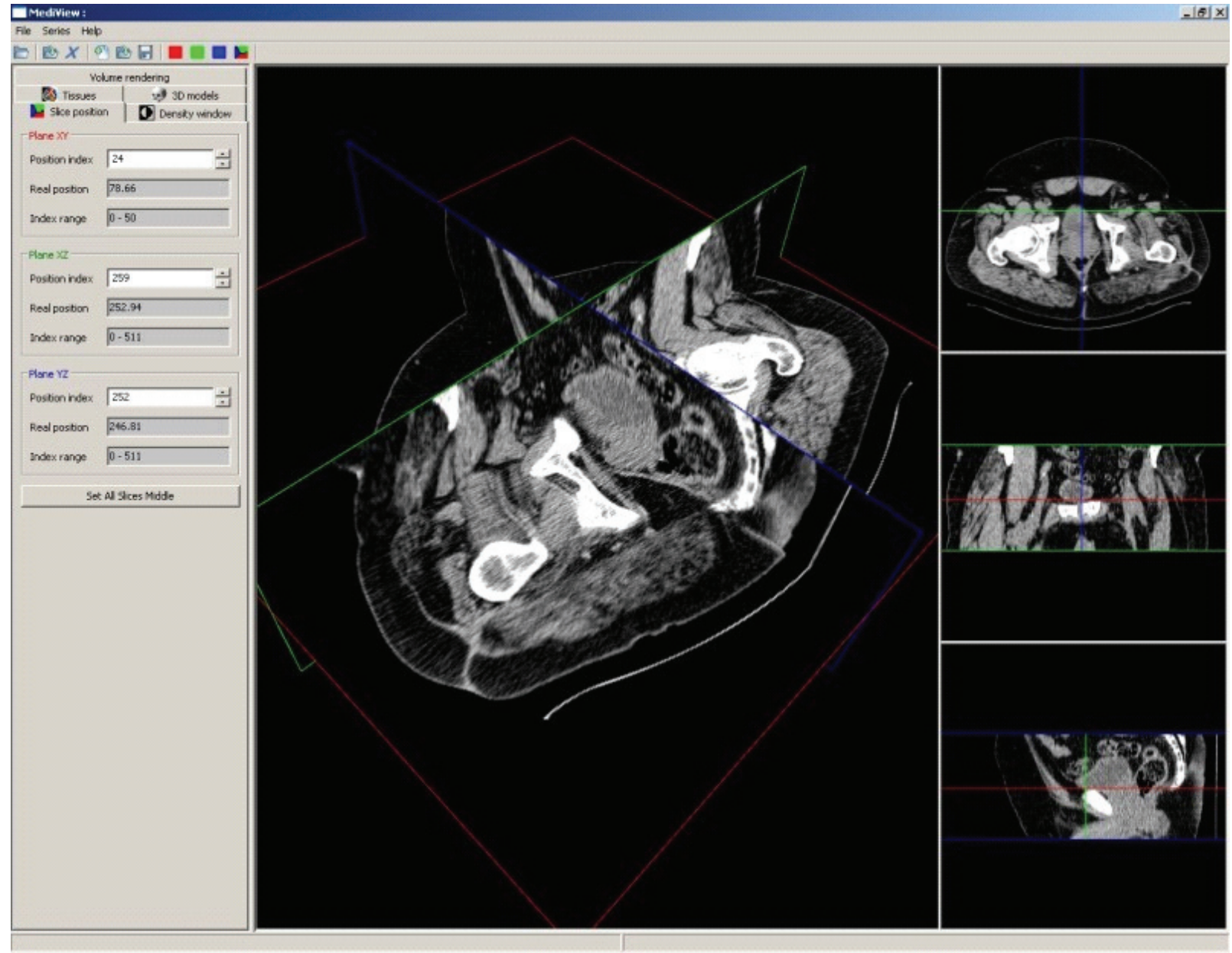

Fig.1. Screen shot during CT data visualisation with MediTools system

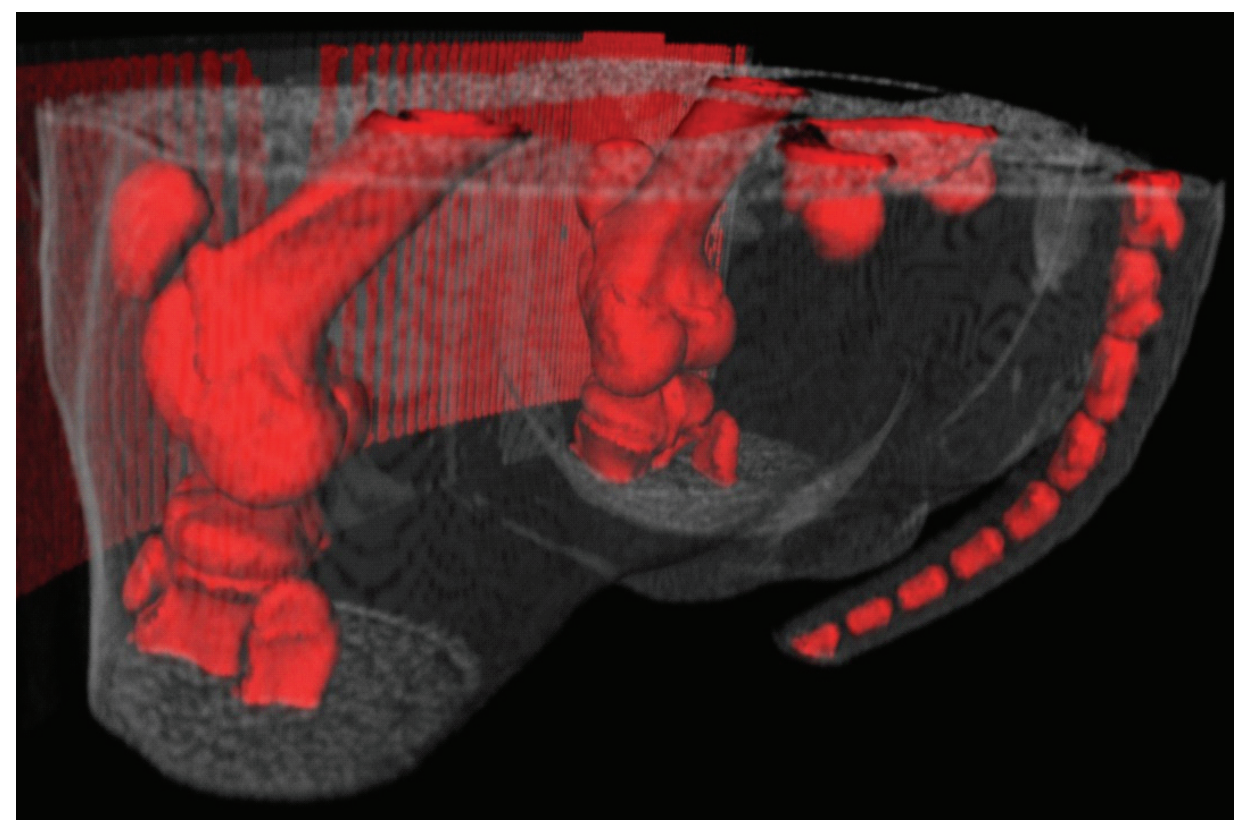

Fig. 2. $\mathrm{CT}$ examination of stifle joints in the miniature pig; bone volume rendering. 
calibration. The pressure transducer was connected to the arterial access port (a.auricularis on contralateral ear than venous access) using extension tubing filled with heparinized saline (200 IU heparin/mL, Heparin, Léčiva, Czech Republic). RR and $\mathrm{ETCO}_{2}$ were logged using side stream with a sensor connected on the tip of the endotracheal tube. $\mathrm{SpO}_{2}$ was sensed with a sensor connected to the patient's tongue. Body temperature was monitored throughout anesthesia.

\section{Surgical procedure}

The surgical procedure was performed on all experimental animals: lateral arthrotomy of the left stifle joint was performed through parapatellar incision. Three circular lesions were created in medial femoral condyle using hand keratome or tubular chisel. The diameter of the most distal lesion was $8 \mathrm{~mm}$, and diameters of two more proximally created lesions were $6 \mathrm{~mm}$. The joints were then flushed with sterile Ringer solution and closed with simple interrupted suture pattern using 2-0 polypropylene (PROLENE, Ethicon). Fascial and subcutaneous layers and skin were closed routinely.

\section{Computed tomography}

All animals underwent $\mathrm{CT}$ examination eight and sixteen weeks after the surgery. The diagnostic imaging procedures were performed on anaesthetized animals. The same anesthetic protocol was used as for surgical procedures.

\section{CT examination}

In this study, CT Philips MX 8000 MX and View workstation were used for all examinations of stifle joints in the experimental animals. CT examination consisted of a series of parallel plane cross section slices and Cartesian discrete volume was created. On the average, 100 slices were performed in each stifle joint. Slice matrix size was $512 \times 512$ voxels with slice thickness of $1 \mathrm{~mm}$. Pixel (voxel) size in the slice plane was $0.5 \mathrm{~mm}$. Therefore, average accuracy of our CT examination was approximately $\pm 0.5 \mathrm{~mm}$ and typical volume size was $512 \times 512 \times 100$ voxels.

All CT examination data were stored in PACS (Picture Archiving and Communication System) system and accessed through metropolitan network (MeDiMed). Therefore, we were able to export CT data in standard DICOM (Digital Imaging and Communications in Medicine).3.0 format. For 3D processing of $\mathrm{CT}$ data and 3D geometrical tissue modelling we used interactive computer graphic system MediTools (Fig. 1) which was formerly developed here (Hamarneh et al. 2005). Among others, this system is specialized for multiplanar 2D- slice displaying and 3D-volume rendering, tissue 3D segmentation and creation of 3D models.

\section{Tissue segmentation}

Input data consisted of 3D discrete volumes with density values. Output data included again 3D discrete volumes, but with segmented tissue indices. For the purpose of our study, we performed segmentation of the tissues in distal femurs in miniature pigs. To separate them, we used simple thresholding or better adaptive thresholding (Lakare 2000). The process of segmentation (Spaněl et al. 2006) consisted of several steps:

- Input CT data were filtered by an anisotropic filter (Wang et al. 1997), which removes noise while preserving well the tissue boundary.

- Densities of bone and cartilage in the stifle joint, which range from 800 to 4000 Hounsfield units (HU) (and thus partly overlap the range of the bone), were precisely tuned for concrete input CT data. Particular parts of cortical bone were separated by some steps of erosion and dilatation filtering (Lakare 2000).

- Parts of cortical bone were removed by manual selecting of tissue densities in the region of stifle joint. All other data were deleted.

- Necessary manual correction and verification of segmented volumes of the distal femur regions were done.

- Cancellous bone areas inside the segmented cortical bones were filled by adapted 3D line seedfill algorithm.

- Necessary manual correction and verification of segmented distal femur volumes were made.

\section{$3 D$ geometrical models}

After finishing the segmentation of selected tissues in the region of examined stifle joint, the tissue 3D model creation process was done. Input CT data were scanned with $1 \mathrm{~mm}$ slice thickness. Slice matrix size was 512 with field of view of $340 \mathrm{~mm}$ diameter. Hence, the voxel size was $1 \times 0.66 \times 0.66 \mathrm{~mm}$. The $\mathrm{CT}$ examination global accuracy was had an average tolerance of $\pm 0.5 \mathrm{~mm}$ for all CT examinations. Discrete data were visualised by methods of volume rendering which did not require segmentation (Fig. 2). The discrete representation was changed to vector representation.

The final creation process of the 3D models of distal femur, which is fully automatic and fast, consisted of several steps: 
A.

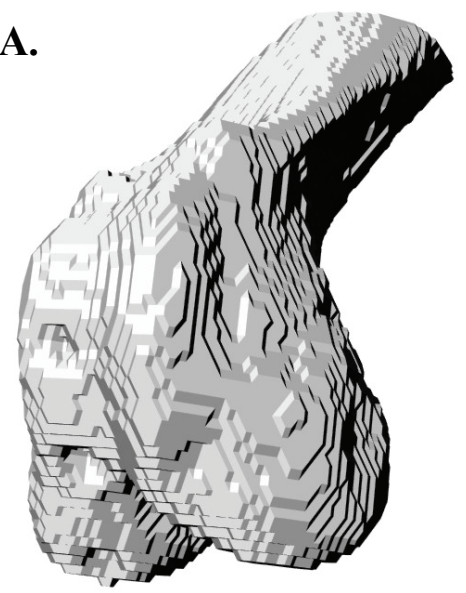

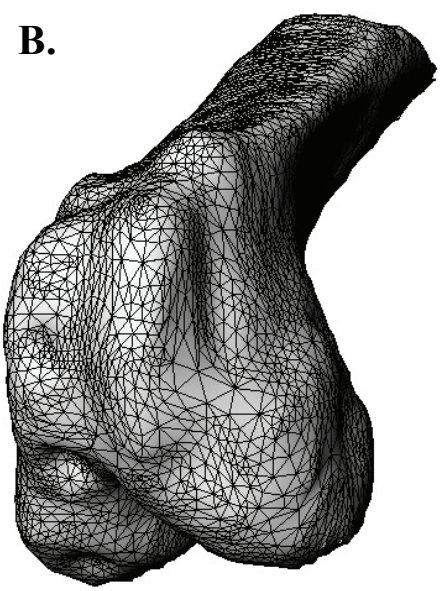

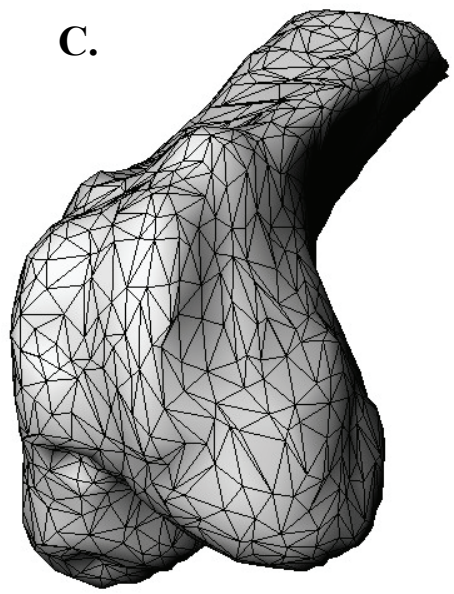

Fig. 2. Process of $3 D$ geometrical model creation of distal femur in the miniature pig (a) vectorised model by the marching-cubes method, (b) smoothed model, (c) reduction of polygon numbers to $10 \%$ of the smoothed model.

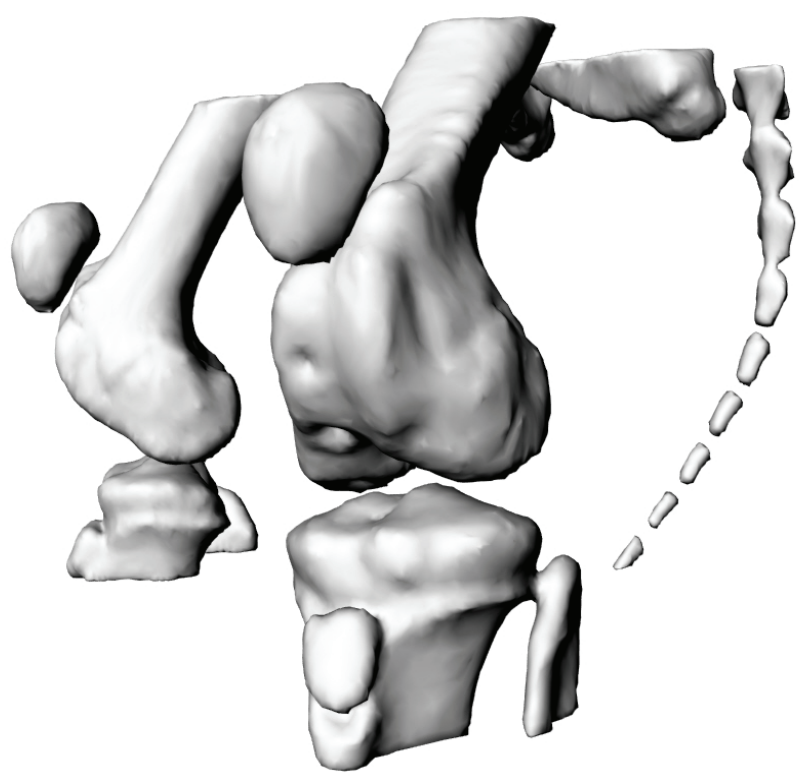

Fig. 3. Computed tomography data based 3D geometrical model of stifle joint in the miniature pig.

- Vectorization of input discrete segmented CT data by the flow-reduction marching- cubes method (Fig. 3a) (Lorensen et al. 1987, Kršek et al. 2005). Smoothing of 3D polygonal boundary model created by theprevious step. The model with sharply demarcated and layered boundary structure due to vectorization of discrete data with given resolution was prepared (Fig. 3b) (Balendran 1999, Taubin 2000).

- Reduction (decimation) of smoothed 3D model polygon numbers by appropriate decimation method (Fig. 3c) (Schroeder et al. 1992, Garland and Heckbert 1997).

- Export of the 3D model into proper data format (STL, VRML, DXF, etc.) for particular application.

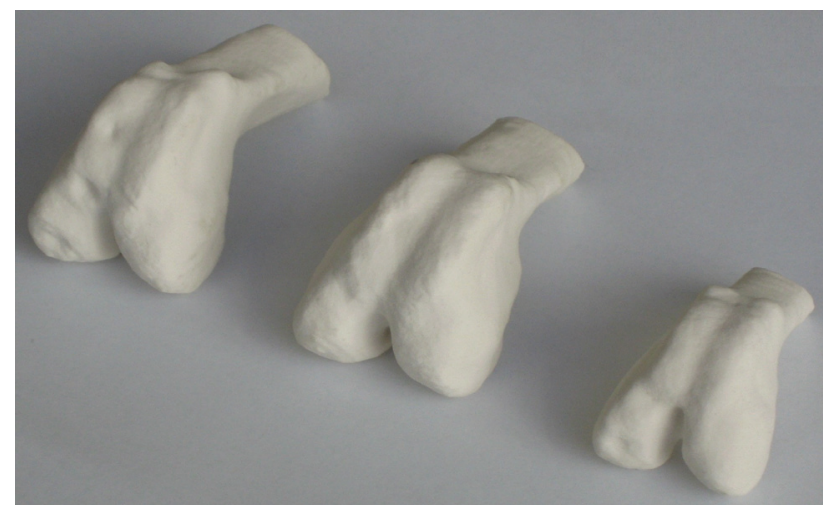

Fig. 4. The real plastic models of distal femurs (large, medium and small - from left to right) with defects of articular cartilage in the miniature pigs.

\section{Real distal femoral plasic models}

Rapid prototyping (RP) technology creating 3D real model directly by a computer, step-by-step from a series of planar parallel thin material layers was used in this study. We used the RP machine Z310 ZCorporation, which creates $3 \mathrm{D}$ real models by printing glue on a plaster layer (Fig. 5).

\section{Results}

\section{Experimental animals}

There were no anesthetic and post-surgery related complications in any of the animals with regard to the surgical procedures (creation of joint surface defects). All wounds healed per primam intentionem. In twenty miniature pigs, we successfully performed $\mathrm{CT}$ examination eight weeks after the surgery. One pig died due to anesthesia complications during the first postoperative CT examination (eight weeks post-op). 
Therefore, sixteen weeks after the surgical procedure we were able to obtain complete $\mathrm{CT}$ data only from nineteen animals.

\section{Tissue segmentation}

For the purpose of this study, we performed segmentation of density values obtained from CT data in the area of the distal femur of miniature pigs. We were able to separate the overlapping density values of soft and hard tissues in the body (cortical bone 1200-1800 HU, cancellous bone 200-1200 HU, cartilage 800-1200 HU, soft tissues 200-400 HU) using simple thresholding or better adaptive thresholding. Cancellous bone density, slightly overlapping the density of soft tissues, was separated using cortical bone density, and thus distinguished from the soft tissues.

A fully automatic process of segmentation was not possible in the study. Our experimental CT data were not ideal and brought about some artifacts and noise. To correct the small discontinuities in segmentation results we had to perform manual correction and verification of automatically created segmentation.

About $85-95 \%$ of the segmentation process was done automatically; the remaining part had to be corrected manually.

\section{$3 D$ geometrical models}

Based on collected CT image data (Fig. 1 and 2), the 3D geometrical models of examined structures in stifle joints (including the geometry of OCD-like lesions) were created (Fig. 3 and 4). Vectorization of input discrete segmented $\mathrm{CT}$ data by the flow-reduction marching-cubes method produced a 3D polygonal (triangular) boundary model of segmented tissues (Fig. 3a). Smoothing of the 3D polygonal boundary model with sharply demarcated and layered boundary structures was successfully done (Fig. 3b), as well as decimation (Fig. 3c). The decimation level was only about $50 \%$. For appropriate smoothing, 10 standard smoothing cycles were applied. Accuracy of the creation process was with an average tolerance $\pm 0.1 \mathrm{~mm}$, with respect to processing parameters for smoothing and decimation. Export of the 3D model into proper data format (STL, VRML, DXF, etc.) for particular application was done without technical difficulties. The time necessary for processing all $39 \mathrm{CT}$ examination data (structure segmentation and 3D models creation) was onn the average $28 \pm 4 \min$ for one examination. In total, $21 \mathrm{~h}$ were necessary for the process of creation of the $3 \mathrm{D}$ geometrical models.

\section{Real distal femoral plastic models}

From the group of 20 miniature pigs, we selected three individuals of different body size to run the model small, medium and large. Earlier created 3D geometrical models were used for construction of the real plastic models of distal femur, showing exact geometry of iatrogenically created OCD-like lesions in the medial condyle of the left femur (Fig. 5). Rapid Prototyping technology ZCorporation was used.

Created real plastic models were made from a plaster composite. Horizontal resolution was 300 DPI and layer thickness was $0.0875 \mathrm{~mm}$. The total creation time for all three models in total was $1 \mathrm{~h}$ and $33 \mathrm{~min}$. The final real plastic models were infiltrated with cyanoacrylate for their surface reinforcement. The accuracy of the creation process was on the average $\pm 0.1 \mathrm{~mm}$.

\section{Discussion}

In this study, we concentrated on 3D geometry modelling of osteochondrosis-like iatrogenic lesions in the medial femoral condyle of miniature pigs. The real plastic models of the distal femur will serve as templates for press-and-fit scaffolds. The scaffold will be made as "anatomical (custom-made) prosthesis" for each experimental animal in future study and will be seeded with mesenchymal stem cells. It will be finally implanted into iatrogenically created OCD-like lesion to enhance healing of this articular cartilage defect. Even though one animal died due to anesthetic complications during the first CT examination eight weeks after the surgery, we were able to collect a very representative sample of CT data necessary for creating the real plastic distal femoral models.

Because the scanned animals were under general anesthesia, our experimental CT examinations have no movement artifacts. The structures of our interest had relatively good contrast when compared to other tissues. Therefore, we did not encounter any problem with tissue segmentation. The whole process could be almost fully automatic and thus was quite fast.

To achieve tissue distribution in the scanned volume, tissue segmentation of input $\mathrm{CT}$ data has to be performed. Source CT data describe the distribution of tissue density (Hounsfield units) in the volume of the scanned body part. Density values of soft and hard tissues in the body do not overlap. For example, the density range for cortical bone is $1200-1800 \mathrm{HU}$, for cancellous bone $200-1200 \mathrm{HU}$, for cartilage $800-1200 \mathrm{HU}$, and for soft tissues 200-400 HU). Simple thresholding or better 
adaptive thresholding (Lakare 2000) was successfully used for their separation by cortical bone density. For input $\mathrm{CT}$ data filtering different smoothing filters (median, Gaussian or anisotropic filtering) can be used. In the study, we used the anisotropic method because we have better experience with anisotropic filtering which removes noise while well preserving tissue boundary (Wang et al. 1997).

Input data for the 3D geometrical model creation process included segmented CT data which had discrete voxel, 3D-based representation. However, boundary (polygonal, vector-based) representation is needed for the creation of our 3D models. Hence discrete representation has to be changed to vector representation. Discrete data were visualized by methods of volume rendering which did not require segmentation (Fig. 2). However, various 3D operations with tissue models still have to be performed: real plastic model creation, scaffold template, surgery simulation, planning, measurements, analysis and data processing. Therefore, the 3D- vector (geometrical) models were necessary for these tasks (Fig. 4).

In three pigs of different size, the 3D geometrical models were used to construct the real plastic models of the distal femoral component of the stifle joint, including iatrogenic geometry of OCD-like lesions. The accuracy of created models (both virtual and real) had average tolerance of $\pm 0.5 \mathrm{~mm}$. It is influenced mainly by the quality of $\mathrm{CT}$ examination. Other processes (3D geometrical models and real plastic-model creation) had the average accuracy of $\pm 0.1 \mathrm{~mm}$. The accuracy of the real plastic models (in average tolerance $\pm 0.5 \mathrm{~mm}$ ) which was achieved in our study is fully sufficient for our purposes and suggested possible applications.

Based on the prepared 3D tissue geometrical models (bone, cartilage etc.) it is possible to create real plastic models of the selected tissues. For the task, it is possible to use two technologies, CNC (computer numeric control) milling and rapid prototyping. $\mathrm{CNC}$ milling technology is thetraditional technology of mechanical engineering for cutting material pieces from used material stock. The main advantages of this technology are the wide range of usable plastic materials, high accuracy $(0.01 \mathrm{~mm})$ and surface quality $(3.2 \mu \mathrm{m})$ level. The main disadvantages are the long milling time, time-consuming and demanding process of $\mathrm{CNC}$ data preparation and geometrical limitations. The technology (5D (5 axes) milling machine AZK HWT D-442 5D $\mathrm{CNC}$ ) is available at our department. However, we prefer the other one.

Rapid prototyping technology creates 3D real plastic models directly by a computer, step-by-step from a series of planar parallel thin material layers. At our department, we have the RP machine Z310 ZCorporation which creates $3 \mathrm{D}$ real models by printing glue on a plaster layer (Fig. 5). The main advantages of this technology are the fully automatic process of real model formation, free geometry without limits, fast forming process (hours). The main disadvantages are accuracy at the level of $0.1 \mathrm{~mm}$, coarse surface quality, limited usable materials (plaster composite, resin etc.). When different material is needed, it is possible to make templates and final models are then cast from the appropriate material (inert Ebalta SG 2000 technical resin, for example).

\section{Conclusions}

This work demonstrates the possibilities and contributions of 3D geometrical modelling in the treatment of stifle joint cartilage injuries. Input data for the processes includes 3D image data from realised CT examinations. Concrete procedure of the construction of 3D geometrical and real plastic models of anatomic structures (femoral component of the stifle joint) were proposed and made. The common procedure was developed and accommodated specifically for the distal femoral model in miniature pigs.

Evaluation of the process was done on experimental CT examinations of twenty animals. On this sample of experimental animals, we optimized our proposed procedure for 3D geometrical and real modeling of joint structures. The created virtual (computer) 3D geometrical models of the distal femur might be further used for more precise evaluation of stifle-joint surface defects, surgery planning, simulation, navigation, or possibly training. In vitro made Real plastic models made in vitro can be used as templates for the press-and-fit collagen scaffold-preparing process. These scaffolds with MSC are expected to be used for articular cartilage-repair enhancement.

The acquired experience will be used in the near future in the next part of our research project dealing with cartilage repair based on press-and-fit implantation of different scaffolds seeded with mesenchymal stem cells into iatrogenically created lesions simulating osteochondrosis of the knee.

\section{Acknowledgements}

This work was supported by the Ministry of Education, Youth and Sport of the Czech Republic (NPV II Research Project 2B06130) and by Grant Agency AGEL-MH. 


\section{References}

BALENDRAN B: A direct smoothing method for surface meshes. In: Proceedings, 8th International Meshing Roundtable, South Lake Tahoe, 1999, 189-193.

BANKMAN IN: Handbook of medical imaging: Processing and analysis, Academic Press, Orlando, FL, 2000.

BURNSTEIN D, BASHIR A, GRAY M: MRI techniques in early stages of cartilage disease. Invest Radiol 8: 409-430, 2000.

ČERNOCHOVÁ P, KANOVSKÁ K, KRŠEK P, KRUPA P: Application of geometric biomodels for autotransplantation of impacted canines. In: World Journal of Orthodontics, Paris, 2005, p 1.

DISLER DG, MCCAULEY TR, KELMAN CG: Fat-suppresed three-dimensional spoiled gradient-echo MR imaging hyaline cartilage defects in the knee: comparison with standard MR imaging and arthroscopy. AJR 167: 127$132,1996$.

GARLAND M, HECKBERT P: Surface simplification using quadric error metrics. In: Siggraph'97 Conference Proceedings, 1997, pp. 209-216.

HALL RK: The role of CT, MRI and 3D imaging in the diagnosis of temporomandibular joint and other orofacial disorders in children. Aust Orthod J. 13: 86-94, 1994.

HAMARNEH G, CHUA V, BORDALO-RODRIGUESB M, SCHWEITZERC M: Deformation Analysis of Hoffa's Fat Pad from CT images of Knee Flexion and Extension. Medical Imaging 57: 527-534, 2005.

HUECK AF, STEIGER P, STOLLER DW, GUER CC, GENANT HK: Quantification of knee joint fluid volume by MR imaging and CT using three-dimensional data processing. J Comput Assist Tomogr 13: 287-293, 1989.

KRAMER J, STIGLBAUER R, ENGEL A, PRAYER L, IMHOF H: MR contrast arthrography (MRA) in osteochondritis dissecans. J Comput Assist Tomogr 16: 254-260, 1992.

KRŠEK P: Flow Reduction Marching Cubes Algorithm. In: Proceedings of ICCVG Springer, Warsaw, 2005, pp 100106.

KRŠEK P, SPANĚL M, ČERNOCHOVÁ P, KANOVSKÁ K, KRUPA P, STOKLAS J, MOLITOR M: 3D Human Tissues Modelling In Clinical Applications. In: Medical Information Visualisation, IEEE CS, GB, 2006.

KRUPA P, KRŠEK P, ČERNOCHOVÁ P, MOLITOR M: 3D real modelling and CT biomodels application in facial surgery. In: Neuroradiology, Springer, Berlin, 2004, p 1.

LORENSEN W, CLINE H: Marching cubes, A high resolution 3D surface construction algorithm. In: Siggraph '87 Conference Proceedings, 1987, pp 163-169.

POTTER HG, LINLATER JM, ALLEN AA, HANNAFIN JA, HAA SB: MR imaging of articular catilage of the knee: a prospective evaluation using fast spin-echo imaging. J Bone Joint Surgery Am 80: 1276-1284, 1998.

RUBENSTEIN JD, LI JG, MAJUMDAR S, HENKELMAN RM: Imaging resolution and signal-to-noise ratio requirements for MR Imaging of degenerative cartilage defects. AJR 155: 549-553, 1990.

SCHROEDER WJ, ZARGE JA, LORENSEN WE: Decimation of triangle meshes. In: Siggraph'92 Conference Proceedings, 1992, pp 65-70.

SPANĚL M, KRŠEK P: Vector-based Medical Image Segmentation using Adaptive Delaunay Triangulation, In: Proceedings of the Sixth IASTED International Conference on Visualization, Imaging, and Image Procesing, ACTA Press, Palma de Mallorca, 2006, p 6.

TAUBIN G: Geometric signal processing on polygonal meshes. In: Eurographics 2000 State of the Art Report (STAR), 2000.

WANG Y, JIN JS, HILLER J: An adaptive nonlinear diffusion algorithm for image filtering. Imaging 3028: 26-37, 1997.

\section{Corresponding author}

Petr Krupa, Klinika zobrazovacích metod, FN u sv. Anny, Lékařská fakulta Masarykovy univerzity, Pekařská 53, 656 91 Brno, Czech Republic. E-mail: necas@eurosat.cz. 